\title{
Commentary
}

Mind the (inferential) gap: Giles et al (2018) provide no convincing evidence for a causal relationship between 'interceptive timing ability' and arithmetic attainment.

\section{Charles Hulme}

Department of Education, University of Oxford

(word count, main text 994 words) 
Giles et al. (2018) stated "...Piaget proposed that sensorimotor interactions with the environment underpin the development of cognitive representations, including our understanding of number." They appear to argue for a specific causal connection between the development of interceptive timing ability and arithmetic. Here I argue their study provides no convincing evidence for such a causal interpretation.

\section{Summary of the Study}

Giles et al. (2018) presented concurrent correlational data from a sample of 309 children, differing widely in age. They measured 5 sensori-motor abilities: steering (tracing a path using a stylus), aiming (moving a stylus to hit a target that appears in different positions), tracking (following a moving dot using a stylus) postural control (balance) and interceptive timing ability (the child moved a custom-made 1-degree-of-freedom Joystick just in time to intercept a moving target). Teacher ratings of attainment in arithmetic, reading and writing were assessed with 15-point scales. It is regrettable that age-standardized measures of children's arithmetic performance were not used which would have provided objective measures of attainment on an interval scale.

\section{Theory}

The paper suggested that there is a causal connection between the development of sensori-motor skills and the development of arithmetic. However, no clear reason is given for why interceptive timing should be particularly closely associated with arithmetic. The abstract states that "learning how objects move in space and time may underpin the development of related abstract representations (i.e mathematics)". This suggests that their steering or tracking tasks (which both involve following moving displays) should relate to arithmetic just as well as interceptive timing. This is an example of where having a preregistered analysis plan would have been particularly helpful. Analyses of their data (https://osf.io/u9ac3/) show that the correlations between arithmetic and interceptive timing, 
steering, aiming and tracking (with each variable partialled for age) are ( $r h o=.20,-.25,-.26$ and -.35 , respectively). Hence, interceptive timing ability is no stronger, and possibly a weaker, correlate of arithmetic than these other motor tasks.

Problems with the design, presentation, analysis and interpretation of data

In any study of individual differences, descriptive statistics and reliabilities should be presented. No such measures are presented in the paper which renders the later analyses difficult to interpret. Measures of poor reliability will have limited correlations with other related variables meaning that differences in reliability between tasks can produce artifactual differences in the pattern of predictive relationships (Cole \& Preacher, 2014). Furthermore, the use of a concurrent correlational design is a very poor way of assessing causal theories (Davis, 1986).

The sample differs widely in age. After controlling for age the authors reported a weak correlation ( $r=.208)$ between interceptive timing ability and arithmetic (they urged caution in interpreting this correlation since their measure of arithmetic is on an ordinal scale; nevertheless, it is clear that any relationship is weak- see the essentially identical Spearman correlation above). Arguably, treating age as a nuisance variable in their analyses is unfortunate. It would have been preferable to assess whether any relationships between sensori-motor abilities and arithmetic vary as a function of age. Their claim (that early variations in sensori-motor learning provide the foundation for later learning about "abstract representations (i.e mathematics)") suggests a stronger relationship might exist earlier, rather than later, in development. Strikingly, in the sample of 124 children aged less than 8 years in their sample there is essentially no relationship between interceptive timing ability and arithmetic after controlling for age ( $r$ ho with both variables partialled for age $=.063[95 \%$ bootstrapped CI -.10,.23]). Based on their own analyses, and those reported here there seems considerable doubt as to whether interceptive timing is even a weak correlate of 
arithmetic skill, and there is no evidence that it is a better a correlate of arithmetic than their other motor tasks.

\section{Thinking clearly about causes}

Leaving these detailed concerns aside, even if variations in interceptive timing are a weak concurrent correlate of arithmetic skills, how should we interpret such a correlation? There are three possibilities: 1 . There is a causal connection (motor skills -> arithmetic) 2 . There is the opposite causal connection (arithmetic -> motor skills), 3. There is no direct connection between the two variables - the correlation reflects some other (unspecified) factor that affects both measures. The authors argued against option 2 as follows "it is difficult to see how enhanced mathematics ability could have improved performance on the interceptive-timing task “. That argument seems reasonable but in the absence of any clearly articulated theory, and with only concurrent data, both interpretations 1 and 2 above seem equally implausible.

Giles et al., conclude there is likely to be a causal relationship between interceptive timing and arithmetic "We should not neglect the importance of sensorimotor development in young children... the present work complements reports that physical activity can exert positive benefits on cognitive processing, even if the mechanisms remain opaque...the quality of early sensorimotor interactions with the environment may have important implications for children's education".

In contrast, I would argue that the weak association between interceptive timing ability (and their other motor measures) and arithmetic is likely to reflect one or several unmeasured factors related to both skills. The paper ignores theories of arithmetic development and current knowledge of the predictors of individual differences in arithmetic development (see e.g. Gilmore, Goebel \& Inglis, 2018). There are a host of cognitive skills 
that are predictors of variations in arithmetic (for example, knowledge of Arabic digits, counting ability and executive function). Many unmeasured factors could explain the very weak concurrent associations between interceptive timing, other motor measures, and arithmetic shown in this paper.

In conclusion, it is crucial to think clearly about causal mechanisms when trying to understand variations in children's educational attainment. There is no reason to believe that the weak concurrent association between interceptive timing skills and arithmetic reported by Giles et al. reflects a causal effect. It would be a great disservice to the field if this work encouraged people to believe that variations in interceptive timing skill are a cause of later developing individual differences in arithmetic.

\section{References}

Cole, D. A., \& Preacher, K. J. (2014). Manifest variable path analysis: Potentially serious and misleading consequences due to uncorrected measurement error. Psychological Methods, 19(2), 300-315. doi: 10.1037/a0033805.

Davis, J.A. (1986). The logic or causal order. Sage

Giles, O.T., Shire, K.A., Hill, L.J.B, Mushtaq, F., Waterman. A., Holt, R.J., Culmer, P.R., Justin H. G. Williams, J.H.G., Wilkie, R.M., \& Mon-Williams, M. (2018). Hitting the Target: Mathematical Attainment in Children Is Related to Interceptive-Timing Ability. Psychological Science, doi.org/10.1177/0956797618772502.

Gilmore, C., Goebel, S.M. \& Inglis, M. (2018). An introduction to mathematical cognition. London: Routledge. 